\title{
Adolf Hurwitz, Geometer
}

\author{
Klaus Volkert
}

Eingegangen: 10. Mai 2020 / Angenommen: 6. Juni 2020 / Online publiziert: 17. Juli 2020

(C) Der/die Autor(en) 2020

Zusammenfassung In diesem Beitrag wird die Probevorlesung vorgestellt, die Adolf Hurwitz anlässlich seiner Habilitation 1882 gehalten hat. Er gibt darin einen bemerkenswerten Überblick zur Lage der Geometrie zu dieser Zeit.

Am 13. Mai 1882 hielt in Göttingen ein junger vielversprechender Mathematiker seine Probevorlesung im Rahmen der Habilitation. Es handelte sich um Adolf Hurwitz (Abb. 1; 1859-1919), der am 26. März 1882 seinen 23. Geburtstag begangen hatte. Der Titel des Vorlesung lautete: „Über die Methoden der neueren Geometrie“. ${ }^{1}$ Den heutigen Leser mag das Thema erstaunen, bringt man doch Hurwitz vor allem mit analytischer Zahlentheorie und Funktionentheorie in Verbindung. Das Interesse an der Geometrie erklärt sich neben der Tatsache, dass Hurwitz ein sehr vielseitiger Mathematiker gewesen ist, aus seinem Werdegang. Hurwitz hatte in Hildesheim den Realschulzweig des Andreanums besucht, wo Herrmann Caesar Hannibal Schubert sein Mathematiklehrer wurde. In seinem, der Dissertation beigefügte Lebenslauf schreibt Hurwitz ${ }^{2}$ :

„In Tertia erhielt ich bei Herrn Oberlehrer Dr. Schubert (jetzt an der Gelehrtenschule des Johanneums in Hamburg) Unterricht in der Mathematik, und seinem

\footnotetext{
1 Das von Hurwitz handschriftlich verfasste Originalmanuskript findet sich im Hochschularchiv der ETH Zürich: ETH-Bibliothek Hochschularchiv Hs 592: 97. Ich danke Nicola Oswald (Wuppertal) für den Hinweis auf diese Quelle, sie und Jörn Steuding (Würzburg) haben mir viele Informationen zu Hurwitz zukommen lassen. Schließlich danke ich Frau Evelyn Boesch vom Hochschularchiv der ETH für die Beschaffung des Textes.

Zu den Einzelheiten von Hurwitz' Habilitation vgl. man Tobies [9, S. 209-210].

2 Vgl. https://www.ma.tum.de/de/fakultaet/hurwitz-gesellschaft.html (letzter Aufruf am 12.05.2020).
}

K. Volkert (ه)

Bergische Universität Wuppertal, Wuppertal, Deutschland

E-Mail: klaus.volkert@math.uni-wuppertal.de 
Abb. 1 Adolf Hurwitz in jungen Jahren (ETH Zürich e-pics)

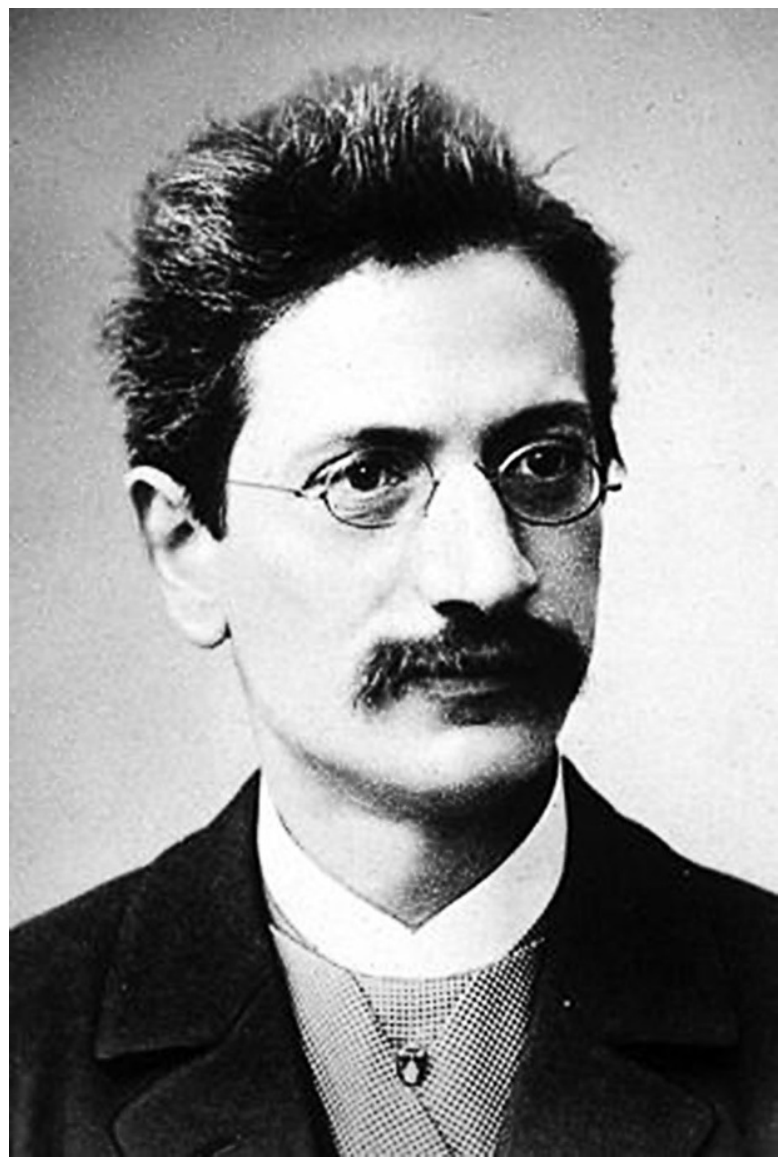

anregenden Einfluss war es zu verdanken, dass bald die Mathematik mein Lieblingsfach wurde. Von Secunda ab trat Herr Schubert mit mir in persönlichen Verkehr und führte mich in die neuere (synthetische) Geometrie ein, vor allem aber auch in seine eigenen Untersuchungen über abzählende Geometrie, die mich bald zu selbständigem Forschen anregten.“

Aus der Zusammenarbeit mit Schubert ergab sich Hurwitz’ erste Publikation „Ueber den Chaslesschen Satz $\alpha \mu+\beta v^{\text {“ }}$ - verfasst zusammen mit seinem Lehrer, wobei aber Hurwitz' Anteil eigens hervorgehoben wird. Ostern 1879 ging Hurwitz nach bestandenem Abitur nach München, um bei F. Klein an der Technischen Hochschule zu studieren. Das geschah - wie Hilbert berichtet ${ }^{3}$ - auf Anregung seines Lehrers und entsprach durchaus dem, was zu einem Absolventen einer Realanstalt passte. In diesem Jahr veröffentlichte Hurwitz seine erste eigenständige Arbeit, wieder zu einem geometrischen Thema „Ueber unendlich vieldeutige geometrische Aufgaben,

\footnotetext{
3 Vgl. Hilbert [3, S. 161].
} 
insbesondere über die Schließungsprobleme“" . Er blieb nur ein Semester, in dem er bei Klein Zahlentheorie hörte, um sich dann für drei Semester nach Berlin zu begeben - ins Mekka der damaligen Mathematik. In Berlin war die Konkurrenz groß und Hurwitz war sehr jung - meistens wechselte man nach Berlin erst in höheren Semestern ${ }^{5}$ - aber dennoch vermochte er im Mathematischen Verein zu beeindrucken:

„Ich erinnere mich noch wie heute, mit welcher Auszeichnung der erst 18jährige Hurwitz, der uns gegenüber ja noch ein Fuchs war (...), von seinen älteren Kommilitonen aufgenommen wurde, als diese sahen, mit welchem Ernste und mit welcher Überlegenheit er in das wissenschaftliche Leben des Vereins eingriff, das gerade zu jener Zeit besonders rege war."6 [8, S. 859].

Wenig überraschend sprach Hurwitz im Mathematischen Verein über geometrische Themen: „Ueber Schliessungsprobleme“"7, „Ueber geometrische Verwandtschaften“" und „Ueber Charakteristiken-Theorien“.

1879 kehrte Hurwitz nach München zurück, musste allerdings im Sommersemester 1880 krankheitsbedingt pausieren. Von München ging er 1880 mit Klein nach Leipzig. Dort promovierte er 1881 mit einer Arbeit über elliptische Modulfunktionen, hatte also die Hinwendung zur Funktionentheorie vollzogen. Wie aber kam Hurwitz nach Göttingen? Das lag an einer universitätspolitischen Widrigkeit: In Leipzig durften nur solche Bewerber habilitieren, die das Abitur eines humanistischen Gymnasiums vorzuweisen hatten. Mit Mühe und nach einigen Kämpfen hatte Klein für W. Dyck, ebenfalls ein Absolvent einer Realanstalt, anfangs 1882 eine Ausnahmeregelung durchgesetzt - aber im Falle Hurwitz schien das wohl hoffnungslos. Also musste eine Alternative her. Da eine derartige Vorschrift an preußischen Universitäten nicht existierte, war der Weg nach dort vorgezeichnet - warum also nicht Göttingen, wo Klein selbst ja auch habilitiert hatte? In Göttingen war Hermann Amandus Schwarz, der 1875 vom Polytechnikum Zürich gekommen war, der tonangebende Mann. Da passte Hurwitz' Wende zur Funktionentheorie natürlich gut. Neben Schwarz wirkte Moritz Abraham Stern als Mathematiker in Göttingen. Auch das konnte für Hurwitz nur von Vorteil sein, war Stern doch der erste nicht-konvertierte jüdische Mathematikprofessor im deutschen Reich. Zudem war er Klein seit dessen Göttinger Zeit freundschaftlich verbunden; es ist anzunehmen, dass Klein ihn in Vorbereitung der Hurwitzschen Habilitation vorab einschaltete. Hurwitz, der nie konvertierte, ist in seiner Laufbahn mehrmals mit antisemitischen Vorbehalten konfrontiert worden. ${ }^{8}$ Bei Stern konnte er sich in dieser Hinsicht Unterstützung erhoffen. Aber auch Schwarz selbst wurde Hurwitz empfohlen, zumindest wenn man seiner Frau glaubt: „Kronecker und Klein empfahlen ihm auf's eindringlichste den

\footnotetext{
4 Abgebildet ist ein Ponceletsches Polygon - dem inneren Kegelschnitt umbeschrieben, dem äußeren einbeschrieben.

5 In Mathematik wurden dort gar kaum für Anfänger geeignete Vorlesungen angeboten.

${ }^{6}$ Rudio [8, S. 859]. F. Rudio sollte ein enger Freund von Hurwitz in Zürich werden, wo beide am Polytechnikum wirkten.

7 Die entsprechende Abhandlung ist in Berlin entstanden, datiert ist sie Berlin 18. Dezember 1878.

8 Vgl. Rowe [6], chap. 14. In Leipzig war um diese Zeit der Astronom F.K. Zöllner aktiv, der sich offen antisemitisch äußerte. Ich danke D. Rowe (Mainz) für den Hinweis auf M.A. Stern.
} 
damaligen Göttinger Ordinarius H.A. Schwarz zur Habilitation daselbst, während er selber schon mit dem ältesten Ordinarius M.A. Stern in Korrespondenz getreten war." ${ }^{\text {"9 }}$ [4, S. 6]. Offensichtlich gab es zu diesem Zeitpunkt noch keine Abneigung zwischen Schwarz und Klein.

Hurwitz legte eine funktionentheoretische, von Weierstraß, seinem ,hochverehrten Lehrer", angeregte Arbeit als Habilitationsschrift ${ }^{10}$ vor, die von Schwarz sehr günstig beurteilt wurde. Da die Habilitation in der philosphischen Fakultät angesiedelt war, also auch Nicht-Mathematiker unter den Zuhörern zu erwarten waren, war Hurwitz' Themenwahl sicher geschickt. Tatsächlich waren anwesend: die bereits genannten Schwarz und Stern, der Physiker Eduard Riecke, Freund von Klein aus Göttinger Tagen, Johann Benedikt Listing, Mathematiker und Physiker, sowie Wilhelm Weber, Physiker. Laut Protokoll dauerte Hurwitz Vortrag nur 20 min.

Eine große Herausforderung, mit der sich die Geometrie in der zweiten Hälfte des 19. Jhs. konfrontiert sah, war die Vielfalt geometrischer Gebiete, die das alte fast monolithische Gebäude der Euklidischen Geometrie abgelöst hatte. 1859 veröffentlichten die „Nouvelles annales“11, eine französische Fachzeitschrift, die sich hauptsächlich an die Mathematiklehrer der Vorbereitungsklassen wandte, eine Liste von ,acht Geometrien, die sich untereinander durch verschiedene Logiken unterscheiden." [1, S. 449], darunter beispielsweise die projective und die infinitesimale Geometrie. Dazu kommen noch die Geometrie der Lage (hier im Sinne von Topologie gemeint) und die Geometrie der Disposition (von J.J. Sylvester), deren Entwicklungsstand eine Einordnung nach Meinung des Verfassers noch nicht zuließ. Kurz: Zehn Geometrien gibt es und nicht mehr nur eine.

In seiner Probevorlesung gibt Hurwitz ein interessantes Panorama der Geometrie zu einem Zeitpunkt, zu dem vieles im Umbruch war. Hurwitz selbst nennt vor allem die projective Geometrie, kurz erwähnt er auch die nichteuklidische. Daneben hatte die algebraische, damals meist analytische Geometrie genannt, sich enorm entwickelt - nicht zuletzt durch die Verwendung Riemannscher Ideen, aufgegriffen von A. Clebsch und vermittelt von seinen Schülern wie A. Brill, M. Noether und F. Klein. Die Theorien von A. Cayley und G. Salmon waren im deutschsprachigen Raum bekannt geworden vermöge der deutschen Bearbeitungen der Salmonschen Lehrbücher durch W. Fiedler, auch die Werke italienischer Geometer, vor allem natürlich von Luigi Cremona, waren bekannt und teilweise übersetzt.

Eigentlich mutig von einem jungen Mathematiker, sich dieser Fülle von Material im Versuch, große Prinzipien darin zu benennen, zu stellen. Das macht ein Interesse der Ausführungen von Hurwitz' aus. Man sieht in ihnen aber auch schon sein großes didaktischen Geschick, sie sind immer klar und deutlich, Beispiele helfen dem Verständnis. Hurwitz hat mit großem Einsatz jahrzehntelang Vorlesungen gehalten am Eidgenössischen Polytechnikum in Zürich, es störte ihn auch nicht, für ein breites

\footnotetext{
9 Samuel-Hurwitz 1984, S. 6 zitiert bei Oswald [5, S. 22-23]. In der Dissertation von N. Oswald finden sich im Kapitel zwei viele weitere sehr interessante Informationen zu Hurwitz' Leben und Karriere.

10 „Ueber die Perioden solcher eindeutiger 2n-fach periodischer Functionen, welche im Endlichen überall den Charakter rationaler Functionen besitzen und reell sind für reelle Werthe ihrer n Argumente" (Journal für die reine und angewandte Mathematik 94 (1883), 1-20).

11 Anonym [1]. Eine deutsche Übersetzung der Liste findet sich in Volkert [12, S. 10].
} 
Publikum einschließlich zukünftiger Ingenieure über Differential- und Integralrechnung zu lessen und dabei Nachsicht zu üben. Davon zeugen viele Vorlesungsskripte von Hurwitz, die sich im ETH-Hochschularchiv befinden. Anders als Freund Minkowski sah er es als Herausforderung an, auch weniger begabte oder interessierte Studierende zu unterrichten und zu fördern. ${ }^{12}$

Die Kernpunkte in Hurwitz' Vortrag sind, wie wir sehen werden: Allgemeinheit, organischer Aufbau und Einheitlichkeit gestiftet durch die neuen Methoden; die Werkzeuge hierfür sind Abbildungen, insbesondere die Projektionen, und Erzeugungsprinzipien im Sinne von Jakob Steiner; mit einem längeren Zitat dieses großen Geometers schloss Hurwitz seinen Vortrag. All das präsentiert er in großer Klarheit und bester Verständlichkeit in seinem kleinen „Meisterwerk der Darstellungskunst" ${ }^{13}$. Interessant ist ein Vergleich mit der historischen Einleitung ${ }^{14}$, die Hankel seinem Lehrbuch der projektiven Geometrie voranstellte, vermutlich eine Quelle, die Hurwitz kannte. Dabei fällt auf, wie präzise Hurwitz die Dinge auf den Punkt bringt und wie sachlich er bleibt.

Hurwitz blieb noch zwei Jahre in Göttingen als Privatdozent, um dann auf ein neu eingerichtetes bezahltes Extraordinariat in Königsberg zu wechseln. Dieses hatte F. Lindemann bei seiner Berufung ausgehandelt und F. Klein hatte den passenden Personalvorschlag. In Königsberg entstand das bemerkenswerte Trio Hurwitz-Hilbert-Minkowski, das fortan in verschiedenen örtlichen Konstellationen sein Wirken ${ }^{15}$ entfalten sollte. 1892 folgte Hurwitz einem Ruf als Nachfolger von G. Frobenius auf die erste Lehrstelle für höhere Mathematik in deutscher Sprache ans Polytechnikum in Zürich, wo er bis zu seinem Tod 1919 wirkte.

\footnotetext{
12 Zu Minkowski vgl. man seine Briefe an Hilbert aus der Züricher Zeit (1896-1902) in Rüdenberg und Zassenhaus [7, S. 85-151].

13 Hilbert [3, S. 167].

14 Hankel [2, S. 1-33].

15 Dieses in der Mathematikgeschichte wohl einmalige Trio ist bislang leider nur partiell, vor allem durch die Veröffentlichung der Briefe von Minkowski an Hilbert, aufgearbeitet worden.
} 


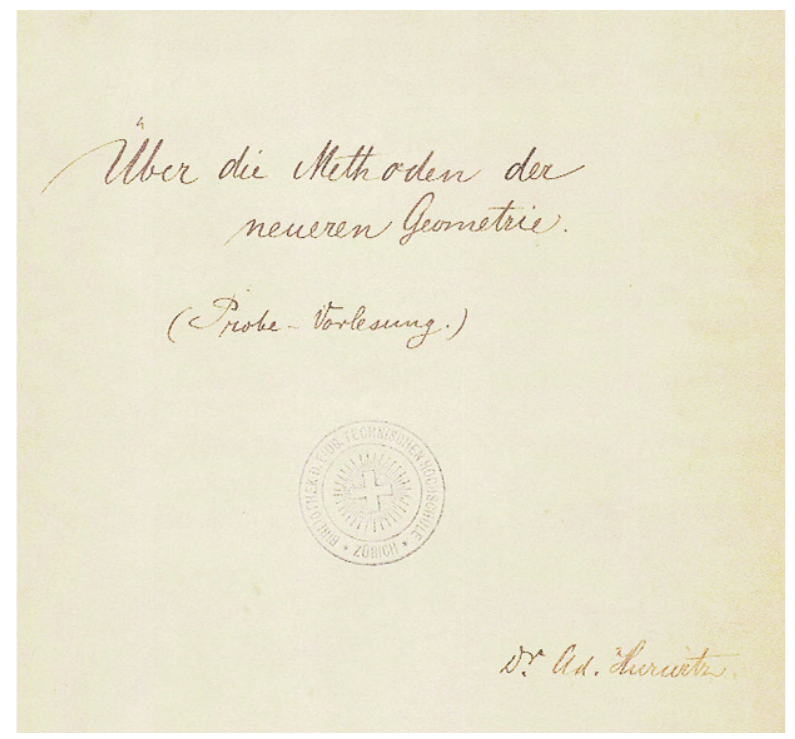

Offensichtlich handelt es sich um das Manuskript, das Hurwitz für seinen Vortrag tatsächlich verwandte. Das zeigen u. a. die darin verwandten Abkürzungen, Randbemerkungen und Hervorhebungen durch farbiges Unterstreichen. Die Schreibweise und Zeichensetzung des Originals wurden in der untenstehenden Transkription beibehalten.

\section{Über die Methoden der neueren Geometrie}

Als zu Anfang des 17. Jahrhunderts Descartes die analytische Geometrie schuf, boten sich den Mathematikern eine Reihe von Fragen dar, welche bekanntlich zu der folgenschweren Entdeckung der Differential- und Integral-Rechnung hinführten. Wenn nun auch in dieser Hinsicht die Ideen Descartes' die schönsten Früchte trugen, so erfüllten sie doch andererseits vorab nicht die berechtigten Hoffnungen, welche die reine Geometrie als solche an dieselben knüpfen durfte. Vielmehr war noch immer im Wesentlichen das System Euklid's die mustergültige Geometrie.

Die Wissenschaft war gleich wie ein Dornröschen in dem Zauber der Jahre erstarrt, die Jünger der Wissenschaft waren in ihrem Anblick versunken und keiner hatte den Muth sie zu erlösen und ihr neues Leben einzuhauchen. Gegen Ende des vorigen Jahrhunderts sollte sich der Bann lösen. Es traten in kurzer AufeinanderFolge eine Reihe ausgezeichneter Mathematiker hervor, die gänzlich abstrahirend von den überlieferten Untersuchungs-Mitteln, geometrische Probleme in Angriff nahmen und in Verfolg dieser Probleme eine ganz neue Disziplin, die neuere oder projectivische Geometrie ${ }^{16}$ erschufen. Der große Erfolg aber, von welchem die Un-

16 „Projectivisch“ war eine Wortschöpfung der 1870er Jahre, vgl. etwa den Titel von H. Hankel „Vorlesungen“ [2] und diverse Arbeiten von F. Klein. Erst später bürgerte sich das grammatikalisch richtige „projektiv“ ein. 
tersuchungen dieser Männer begleitet waren, beruht auf einem unterscheidenden Merkmale ihrer Methoden von denen der alten Geometrie.

Während nämlich die letztere für jedes einzelne Problem einen besonderen Ansatz erfordert, giebt die neuere Geometrie eine kleine Zahl von Vorschriften, nach denen die umfassendsten Classen von Problemen gelöst werden können.

Wir wollen versuchen diese Behauptung durch eine nähere Betrachtung der Methoden der neueren Geometrie zu rechtfertigen. Hierbei werden sich meine Ausführungen naturgemäß in drei Abschnitte gliedern, von denen der erste die Methode der Abbildung, der zweite die der Erzeugung und der dritte die der Übertragung des Projectivischen in's Metrische behandeln soll.

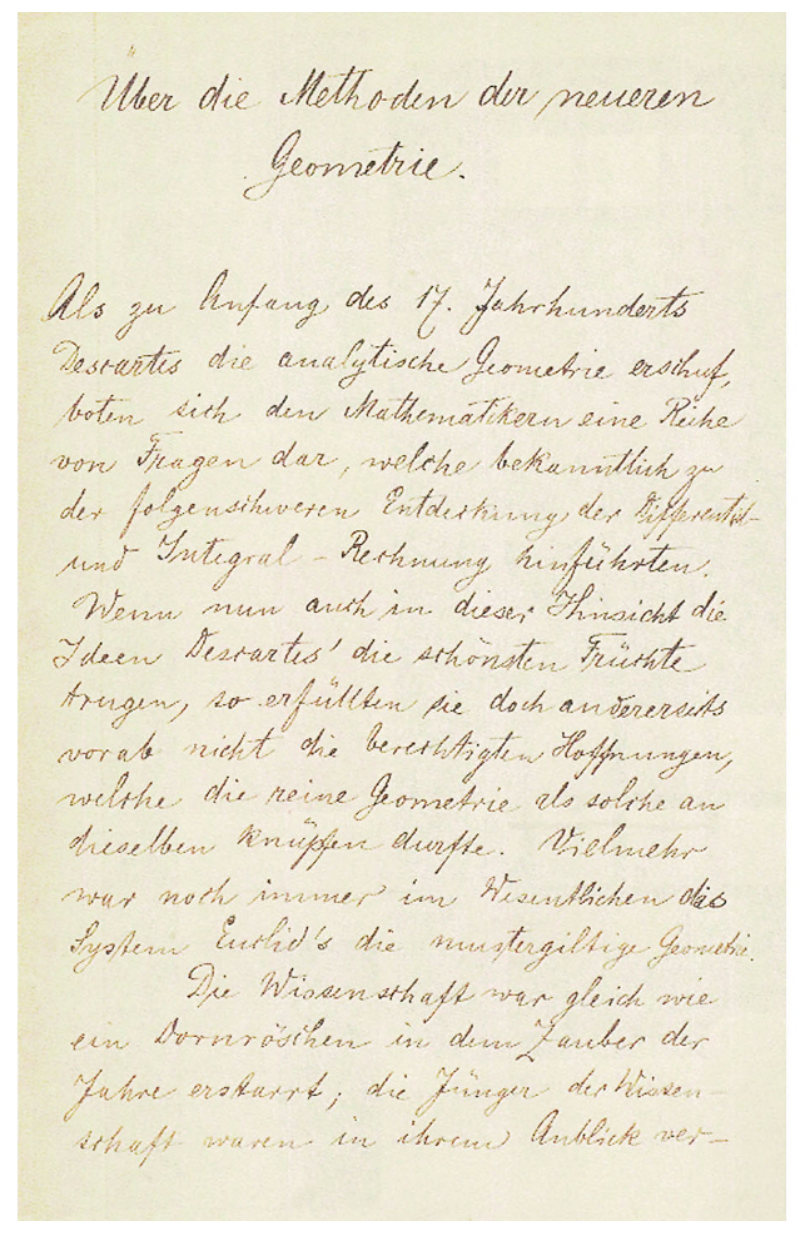

Indem wir uns der Methode der Abbildung zuwenden, soll uns zunächst der specielle Fall dieser Methode, nämlich die Lehre von der Projection, beschäftigen.

Im Jahr 1795 veröffentlichte der französische Mathematiker Monge seine Géométrie descriptive. Hier wurde ein ganz neuer Gedanke vorgetragen, wel- 
Abb. 2 Zeichnung von Hurwitz zur Zentralprojektion

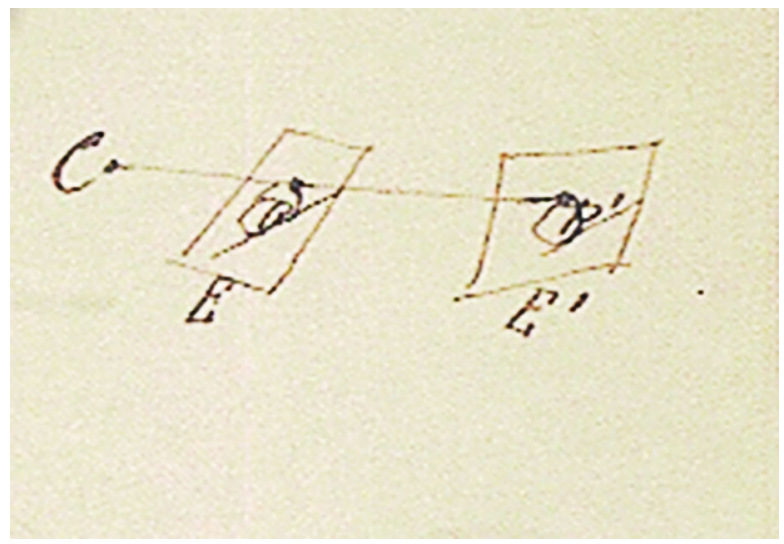

cher ursprünglich aus praktischen Bedürfnissen entsprungen, für die Ausbildung neuer geometr. Theorien bahnbrechend wurde.

Monge lehrte nämlich räumliche Gebilde vollständig durch Zeichnungen darzustellen. Zwei Zeichnungen, der Grund-Riß und der Auf-Riß, das sind die orthogonalen Proj. auf zwei zu einander senkrechte Ebenen, reichen hin, um jedes räumliche Gebilde vollständig zu charakterisieren. ${ }^{17}$ Bei der näheren Ausführung dieses Gedanken ergab sich nun häufig, daß Eigenschaften, die an dem räumlichen Gebilde keines Beweises bedurften andere Eigenschaften der Projectionen zur Folge hatten, die an sich nicht so evident waren. Von diesem Umstand veranlaßt, sah Ponc. ${ }^{18}$ ganz ab von der praktischen Anwendbarkeit der Projections-Methode, um dieselbe zu einem rein theoretischen Hilfsmittel der Untersuchung geometr. Figuren auszubilden. Als russischer Kriegs-Gefangener in Saratow (1812-14) legte er die Grundlagen zu seinen tiefgreifenden Untersuchungen, die 1822 in dem Werke „Traité des propriétés projectives des figures“ zusammen gefaßt erschienen.

Zunächst ersetzt Poncelet die Orthogonal-Projection durch die allgemeinere Central-Projection (vgl. Abb. 2; K. V.).

Man denke sich im Raum einen festen Punkt C, das Projections-Centrum, und zwei Ebenen E und $E^{\prime}$. Dann heißt der Punkt $\mathrm{P}^{\prime}$ der zweiten Ebene die Projection des Punktes $\mathrm{P}$ der ersten Ebene vom Punkte $\mathrm{C}$ aus, wenn $\mathrm{C}$ P $\mathrm{P}^{\prime}$ in gerader Linie liegen. Beschreibt der Punkt $P$ in der Ebene E eine Figur $F$ so beschreibt der Punkt $\mathrm{P}^{\prime}$ entsprechend eine Figur $\mathrm{F}^{\prime}$ in der Ebene $\mathrm{E}^{\prime} ; \mathrm{F}^{\prime}$ heißt die Projection der Figur $\mathrm{F}$.

Poncelet nennt nun alle diejenigen Eigenschaften einer Figur projectivisch, welche sich nothwendig in allen Projectionen dieser Figur wiederfinden. Um ein Beispiel einer solchen projectivischen Eigenschaft anzuführen, sei in der Ebene E eine Curve und eine sie berührende Gerade angenommen, die Projection dieser Figur von irgend einem Punkte aus auf irgendeine zweite Ebene besteht wieder aus einer Curve und einer diese berühr. Geraden. Deshalb wird man also die Berührung

17 Das ist nur dann richtig, wenn das Objekt konvex ist.

18 Lies: Poncelet. 
einer Curve mit einer Geraden als eine projectivische Eigenschaft des aus Curve und Geraden constituirten Gebildes zu bezeichnen haben.

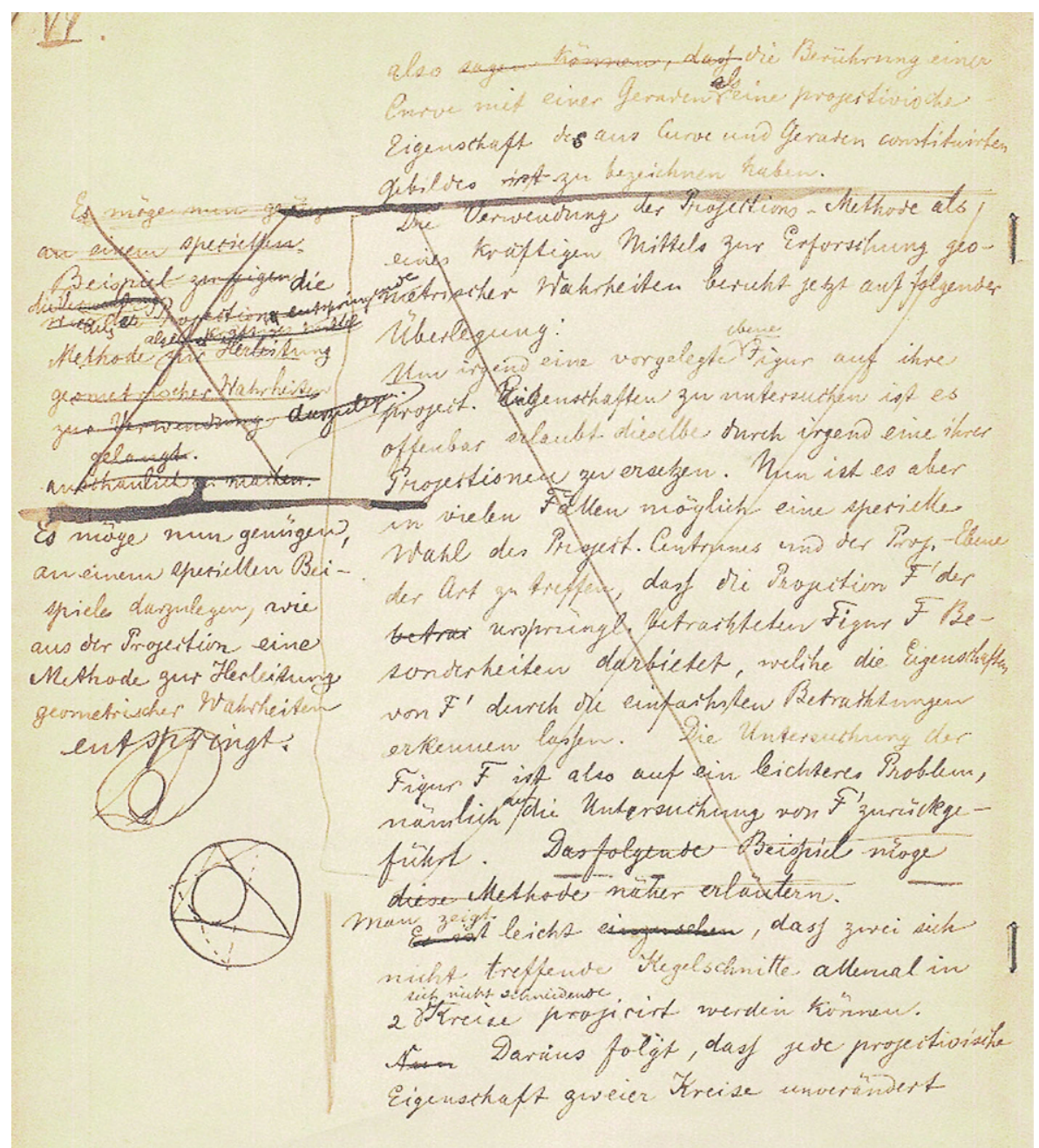

Man zeigt leicht, daß zwei sich nicht treffende Kegelschnitte allemal in 2 sich nicht schneidende Kreise projicirt werden können. Daraus folgt, daß jede projectivische Eigenschaft zweier Kreise unverändert gültig bleibt, wenn wir an die Stelle der Kreise zwei Kegelschnitte setzen. So ergibt sich z.B. aus einer von Euler herrührenden Formel leicht, daß wenn ein Dr. ${ }^{19}$ existirt, welches einem von 2 Kreisen ein-, dem andern umbeschrieben ist, daß dann unzählig vieler solcher Dreiecke gefunden werden können (vgl. Abb. 3; K. V.). Aus der Methode der Projection folgt nun

19 Lies: Dreieck. 
Abb. 3 Figur von Hurwitz zum Schließungsproblem

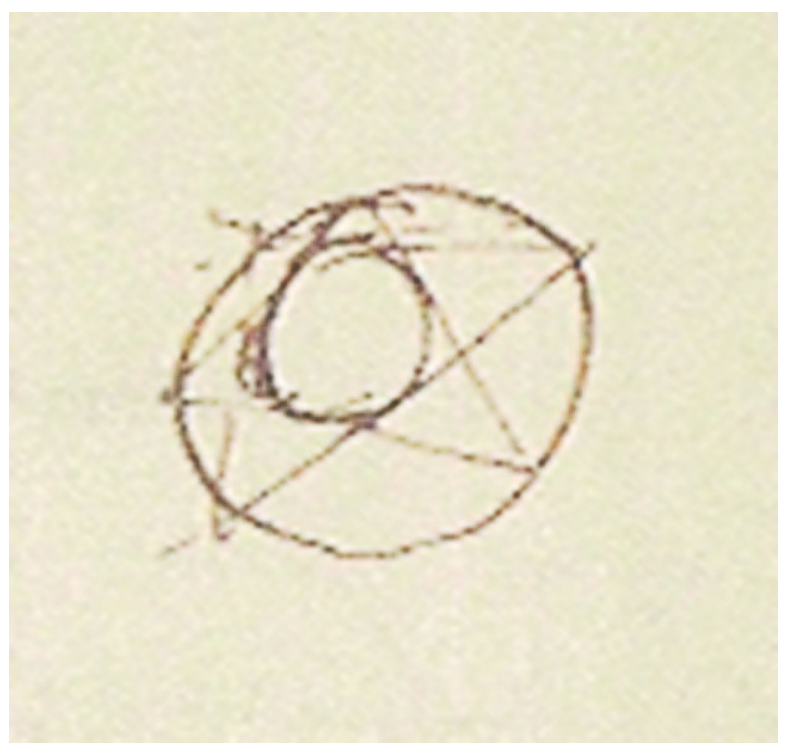

also sofort, daß dieselbe Eigenschaft für zwei sich nicht schneidende Kegelschnitte ausgesprochen werden darf.

Die Methode der Projection hat, was ich nicht mit Stillschweigen übergehen kann, eine Anschauung begründet, welche der modernen Geometrie im Gegenzug zur Euklidischen eigenthümlich ist. Diese Anschauung ist die Auffaßung des Parallelismus als einer speciellen Form des Schneidens (vgl. Abb. 4; K. V.). Der Ausspruch: parallele Geraden einer Ebene schneiden sich in einem unendlich fernen Punkte, bedeutet weiter nichts, als daß eine Schaar paralleler Geraden durch Projection in eine andere Schaar durch einen Punkt laufende Geraden verwandelt werden kann (,kann“ wohl versehentlich durchgestrichen; K. V.).

Ähnlicherweise ist der Ausdruck zu verstehen, daß die unendlich fernen Punkte einer Ebene in gerader Linie liegen. Diese Bedeutung der modernen Ausdrucksweise des Parallelismus ist beiläufig bemerkt in neuerer Zeit häufig verkannt worden, so daß gelegentlich darüber gestritten wurde, ob die unendlich ferne Gerade wirklich existire oder ob sie ein eingebildetes Ding sei. ${ }^{20}$ Die Entscheidung ist nach dem Gesagten nicht zweifelhaft.

Der Fortschritt von der Projection zu dem allgemeinen Begriff der Abbildung hat sich verhältnismäßig langsam und durch verschiedene Zwischen-Glieder vollzogen. Erst Möbius hat in seinem klassischen Werke „,der barycentrische Calcul“21 den Begriff der Abbildung in allgemeiner Weise aufgefaßt. Verstehen wir der Kürze halber unter einem geometr. System eine gesetzmäßig bestimmte Gesamtheit von endlich oder unendlich vielen geometr. Gebilden und nennen wir jedes einzelne Ge-

\footnotetext{
20 Hurwitz spielt hier auf eine hitzige Diskussion in den ersten Bänden der ,Zeitschrift für den mathematischen und naturwissenschaftlichen Unterricht“ an, die einiges Aufsehen erregte. Vgl. Volkert [11].

211827 erschienen.
} 
Abb. 4 Verwandlung einer Schar von Parallelen in eine Schar von sich in einem Punkte schneidenden Geraden

bilde ein Element des Systems, so können wir die allgemeine Abbildungs-Methode folgendermaßen charakterisieren:

Man denke sich daß in irgend welcher gesetzmäßiger Weise die Elemente eines geometr. Systems auf die Elemente eines zweiten bezogen seien, so darf einem Elemente des ersten Systems ein oder mehrere (auch unendlich viele) Elemente des zweiten entsprechen. Dann wird man aus den Eigenschaften der Gebilde des ersten Systems Sätze für die entsprechenden Gebilde des zweiten Systems herleiten können. Unter dieses allgemeine Schema der Abbildung fallen außer der ProjectionsMethode erstens die Theorie der Polaren von Curven und Flächen, welche die Punkte einer Ebene des Raumes auf Curven- bzw. Flächen-Schaaren abbildet; ferner das Princip der Dualität, welches aus einer gewissen Abbildung der Punkte des Raumes auf die Ebenen des Raumes entspringt; hierher gehört (weiter) die Untersuchung alg. Flächen durch ihre Abbildung auf die Ebene, wobei die Fläche 2. Ordn. ein sehr interessantes Bespiel liefert.

(Randbemerkung. Ich muß darauf Verzicht leisten weitere Beispiele von Abbildungs-Methoden anzuführen.)

Hat so die Methode der Projection durch ihren begriffl. Kern zu weit allgemeineren Methoden hingeführt, so hängt mit ihr aufs Innigste auch die Idee der Erzeugung zusammen, deren Einführung in die Geometrie eins der unsterblichen Verdienste des großen Jacob Steiner's ist. Wenn wir in einer Ebene irgend eine Gerade G und einen Punkt S haben, so ist die denkbar einfachste Vorstellung, wenn man jeden Strahl durch S den auf ihm liegenden Punkt von P [Schreibfehler: sollte G heißen] zuweist. Man sagt dann, daß die Gerade $G$ und das Strahlbüschel S perspect. ${ }^{22}$ [vgl. Abb. 5; K. V.]. liegen oder persp. aufeinander bezogen sind. Desgleichen heißen zwei Gera-

22 Lies: perspectivisch. 
Abb. 5 Zeichnung von Hurwitz zu projectiv liegenden Geraden

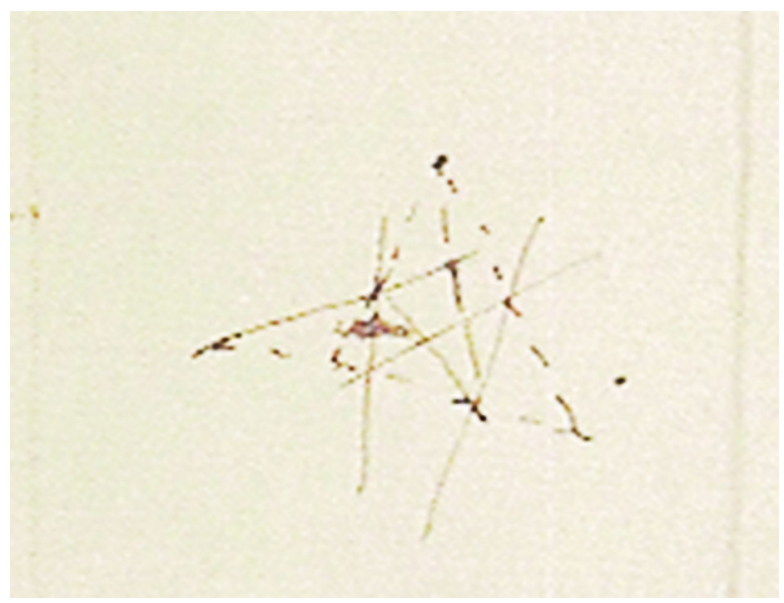

den perspectivisch, wenn sie zu demselben Strahlbüschel, zwei Strahlbüschel, wenn sie zu der selben Geraden perspectivisch sind. Liegen in einer Reihe von Geraden und Strahlbüschel je 2 aufeinander Glieder der Reihe perspectivisch, so sagt man, daß zwei beliebige Elemente der Reihe projectiv. aufeinander bezogen sind, die Perspectivität erscheint dabei offenbar als ein specieller Fall der Projectiv. ${ }^{23}$

Hat man nun projective Strahbüschel hergestellt, die nicht perspect. liegen, so erzeugen dieselben durch die Gesammtheit der Punkte in welchen je 2 entsprechende Strahlen sich treffen einen Kegelschnitt (vgl. Abb. 6; K. V.). Auf diese Art ist also in wahrhaft synthetischer Weise der Kegelschnitt ein Gebilde höherer Art aus einfachen Gebilden, den Strahlbüscheln, hergeleitet.

Wie der Kegelschnitt so entstehen analog alle complicirteren geometr. Gebilde aus bekannten einfacheren Gebilden durch einen Erzeugungs-Process und es offenbart sich die große Fruchtbarkeit der Ideen-Bildung in der spielenden Leichtigkeit, mit welcher sich die Eigenschaften complicirter Gebilde aus den Eigenschaften der sie erzeugenden Gebilde ergeben. So sind z.B. die Sätze über Kegelschnitte, welche Desargues, Pascal, Brianchon und andere mit künstlichen Mitteln entdeckten, ein unmittelbarer Ausfluß der erwähnten Erzeugungs-Weise der Kegelschnitte.

Es darf aber ein Mangel dieser Methode nicht verschwiegen werden, da sie nämlich die Gebilde synthetisch entwickelt, so bleibt sie immer beim Speciellen stehen. Allgemeines, wie z.B. Eigenschaften aller algebr. Curven, ist ihr nur schwer zugänglich.

Der einzige Weg, der hier zum Ziele führen kann, ist der Schluß von $n$ auf $n+1$ : Ein allgemeiner Satz ist bewiesen, wenn man zeigt daß derselbe für Curven 1. Grades, d.h. für die gerade Linie, gilt und für Curven $n+1^{\text {ter }}$ Ordnung richtig ist, wenn er für Curven $\mathrm{n}^{\text {ter }}$ Art als bewiesen vorausgesetzt wird. Diese Beweismethode ist jedoch einigermaßen beschwerlich und kann sich nicht messen mit den bequemen analytisch-geometrischen Methoden, die von Monge, Bobillier, Plücker, Hesse u. anderen ausgebildet sind.

23 Lies: Projectivität. 
Abb. 6 Zeichnung von Hurwitz zur Erzeugung eines Kegelschnitts durch projektive Strahlenbüschel

Die Idee der Erzeugung ergiebt bei consequenter Durchführung einen vollkommen organischen Aufbau der Geometrie; alles entwickelt sich aus wenigen einfachen Betrachtungen, welche die Keime aller geometrischen Sätze ausmachen. In größerer methodologischer Vollendung und Reinheit als bei Steiner findet sich eine entsprechende Darstellung der Geometrie bei von Staudt. In seinen Schriften „Geometrie der Lage“" 24 und Beiträge zur Geom. der Lage ${ }^{25}$ baut von Staudt die Geometrie ganz auf Anschauung, indem er den Begriff der Größe vollkommen aus seiner Darstellung verbannt. Jedoch treten dabei in den Elementen Schwierigkeiten auf über die mehrfach discutirt worden ist, aber die wie es scheint noch nicht von allen Mathem. als erledigt angesehen werden. ${ }^{26}$ Wenn es nun anfangs den Anschein hatte, als sollten der neueren Geometrie alle Sätze, die sich auf Größen-Verhältnisse beziehen, unzugänglich bleiben, so zeigte sich später vielmehr, daß die ganze Lehre der Metrik nur ein specieller Fall der projectivischen Geometrie bildet. Diese Auffaßung und die damit verbundene Methode zur Herleitung metrischer Sätze aus projectiv. und umgekehrt, gilt als eine der schönsten Leistungen des französ. Mathemat. Michel Chasles.

Es ist ein auffallendes Paradoxon, daß zwei Kugeln eine Durchschnitts-Curve 2. Grades, nämlich einen Kreis und nicht, wie zwei Flächen 2. Grades im Allgemeinen eine Durchschnitts-Curve 4. Grades bestimmen. Dieses Paradoxon löst sich in

\footnotetext{
241847.

25 In drei Heften 1856-1860.

${ }^{26}$ Es geht hier um so genannte Stetigkeitsannahmen, die von Staudt beim Beweis des Fundamentalsatzes der projektiven Geometrie verwendet hatte, Klein hatte hierauf 1872 hingewiesen, was dann eine intensive Diskussion auslöste; vgl. Voelke [10].
} 
der Weise, daß alle Kugeln durch einen unveränderlichen imaginären Kreis gehen, der in der unendlich fernen Ebene gelegen ist und als Kugelkreis bezeichnet wird. ${ }^{27}$

Chasles hat nun gezeigt, daß alle metrischen Eigenschaften einer Figur weiter nichts sind als projectivische Eigenschaften des aus dem Kugelkreis und jener Figur zusammengesetzten Gebildes.

Cayley und andere haben von dieser Auffassung ausgehend eine Geometrie ${ }^{28}$ entwickelt, bei der eine feste Fläche 2. Grades ausgezeichnet wird. Diese Geometrie stimmt im Wesen der Sache mit der sogenannten nicht-Euclid. Geometrie überein. Sie geht über in die gewöhnliche Maß-Geometrie, wenn man die zu Grunde liegende Fläche 2. Grades in den Kugelkreis degeneriren läßt.

In der Vorrede zu Steiners Hauptwerke: „der systematischen Entwicklung der Abhängigkeit geometrischer Gestalten von einander" ${ }^{\prime 29}$, findet sich folgende Stelle: „Wenn Jemand alle bis jetzt bekannt gewordenen Sätze und Aufgaben nach den bisher üblichen Vorschriften beweisen und zu lösen sich vornehmen wollte, so wäre dazu viel Zeit und Mühe erforderlich und am Ende hätte man doch nur eine Sammlung von auseinanderliegenden wenn auch scharfsinnigen Kunst-Stücken aber kein organisch zusammenhängendes Ganze zu Stande gebracht.““

Im Anschluß an diese Worte können wir am besten den großen Erfolg der neueren Geometrie kennzeichnen: Heutzutage bietet es gar keine Schwierigkeiten dar das ganze Gebiet der unzählig vielen geometrischen [Sätze; K. V.] zu beherrschen; denn wer sich die kleine Zahl von fruchtbaren Methoden angeeignet hat, welche die neuere Geometrie aufstellt, der ist im Stande alle jene Sätze ohne Aufwand sonderlichen Scharfsinns zu entwickeln.

Funding Open Access funding provided by Projekt DEAL.

Open Access Dieser Artikel wird unter der Creative Commons Namensnennung 4.0 International Lizenz veröffentlicht, welche die Nutzung, Vervielfältigung, Bearbeitung, Verbreitung und Wiedergabe in jeglichem Medium und Format erlaubt, sofern Sie den/die ursprünglichen Autor(en) und die Quelle ordnungsgemäß nennen, einen Link zur Creative Commons Lizenz beifügen und angeben, ob Änderungen vorgenommen wurden.

Die in diesem Artikel enthaltenen Bilder und sonstiges Drittmaterial unterliegen ebenfalls der genannten Creative Commons Lizenz, sofern sich aus der Abbildungslegende nichts anderes ergibt. Sofern das betreffende Material nicht unter der genannten Creative Commons Lizenz steht und die betreffende Handlung nicht nach gesetzlichen Vorschriften erlaubt ist, ist für die oben aufgeführten Weiterverwendungen des Materials die Einwilligung des jeweiligen Rechteinhabers einzuholen.

Weitere Details zur Lizenz entnehmen Sie bitte der Lizenzinformation auf http://creativecommons.org/ licenses/by/4.0/deed.de.

\footnotetext{
27 Man komplexifiziert also die Situation.

28 Man spricht heute von den so genannten Cayley-Klein-Geometrien.

291832.
} 


\section{Literatur}

1. Anonym: Sur diverses géométries différentes. Nouv Ann Math 18, 445-446 (1859)

2. Hankel, H.: Die Elemente der projectivischen Geometrie in synthetischer Behandlung. Teubner, Leipzig (1875). Hrg. von Harnack, A.

3. Hilbert, D.: Adolf Hurwitz. Math. Ann. 83, 161-172 (1921)

4. Samuel-Hurwitz, I.: Erinnerungen an die Familie Hurwitz, mit Biographie ihres Gatten Adolph Hurwitz, Prof. für höhere Mathematik an der ETH. ETH-Bibliothek Hochschularchiv HS 583a: 2 (1984)

5. Oswald, N.: Hurwitz's complex continued fractions (dissertation Würzburg 2014) (2014). https:// opus.bibliothek.uni-wuerzburg.de/opus4-wuerzburg/frontdoor/deliver/index/docId/10604/file/oswald_ nicola_hurwitz.pdf, Zugegriffen: 29. Mai 2020

6. Rowe, D.: A Richer Picture of Mathematics. Springer, Cham (2018)

7. Rüdenberg, L., Zassenhaus, H.: Hermann Monkowski Briefe an David Hilbert. Springer, Berlin, Heidelberg, New York (1973)

8. Rudio, F.: Adolf Hurwitz. Vierteljahrsschr. Naturforsch. Ges. Zürich 64, 859-861 (1919)

9. Tobies, R.: Felix Klein. Springer Spektrum, Berlin, Heidelberg (2019)

10. Voelke, J.-D.: Le théorème fondamental de la géométrie projective: évolution de sa preuve entre 1847 et 1900. Arch. Hist. Exact Sci. 62, 243-296 (2008)

11. Volkert, K.: Are there points at infinity? In: Bioesmat, L. (Hrsg.) Eléments d'une biographie de l'espace projectif, S. 197-205. PUN, Nancy (2010)

12. Volkert, K.: Das Undenkbare denken. Springer Spektrum, Berlin, Heidelberg (2013) 